\title{
AN EXAMINATION OF THE PLANETESIMAL IMPACT HYPOTHESIS OF THE FORMATION OF CP STARS*
}

\author{
CHARLES R. COWLEY \\ University of Victoria and Dominion Astrophysical Observatory, Victoria, B.C., Canada \\ and University of Michigan, Ann Arbor, Mich., U.S.A.
}

(Received 21 March, 1977)

\begin{abstract}
The impact hypothesis has been found to provide a qualitative basis for an understanding of many of the characteristics of the CP stars. It founders primarily upon the problem of underabundances of certain elements and large variations in isotopic abundances. We conclude, however, that the hypothesis should not be prematurely discarded, since it may be combined with other mechanisms to account for the variety of abundance peculiarities of the $\mathrm{CP}$ stars.
\end{abstract}

\section{Introduction}

The chemically peculiar (CP) stars of the upper main sequence fall into groups or families, each showing typical abundance peculiarities. There is at least a loose connection between the abundance anomalies in the different families and the surface temperatures of the stars, and there are also characteristic chemical distinctions among those stars which have strong surface magnetic fields and those which do not.

Very high dispersion studies of these stars have revealed complex abundance patterns which defy all current efforts at an explanation. Guthrie (1971) divided the $\mathrm{CP}$ star theories into two categories - nuclear and non-nuclear. We shall use the term 'differentiated' rather than 'non-nuclear', since all of the elements were made by nuclear processes, and the abundances resulting from the nucleosynthesis were then modified or differentiated. By implication, if the differentiation has not been severe, the nuclear pattern may still be discovered, and this is a useful concept to keep in mind.

Active work on a nuclear origin of the abundance anomalies reached its peak in the 1960's. Since that time, theories of differentiation have generally been considered more promising. We shall attempt only a brief précis of these ideas here.

Michaud's $(1970,1975)$ diffusion hypothesis is, perhaps, the most favorably regarded of the differentiation schemes. According to this work certain elements are selectively pushed upward into the stellar photospheres by radiation pressure acting either on absorption lines or continua. Other elements, whose lines or continua are not favorably located, sink beneath the observable outer layers of the star. Havnes and Conti (1971, see also Havnes, 1975) proposed an alternate scheme in which elements could be selectively accreted from the interstellar medium with the help of stellar magnetic fields.

* Contributions from the Dominion Astrophysical Observatory, Victoria, B.C., No. 342-NRC No. 16101. 
Review articles on the CP stars are available by Sargent (1964), Preston (1974), and the Jascheks (1974); the proceedings of IAU Colloquium No. 32 on the Physics of Ap Stars provides voluminous up-to-date introductory and background material. Recently, we wrote a popular introduction to the puzzle of the CP stars (Cowley, 1976a, b).

The purpose of the present article is to explore one notion which has appeared from time to time in the literature, but has rarely been given detailed consideration. This is the idea that the abundance anomalies in upper Main-Sequence stars are due to the infall of planets or planetesimal bodies. We shall call it the 'impact hypothesis.'

For the most part, the impact hypothesis of CP star anomalies has been discussed in the Russian literature, and important articles have not been translated (cf. Dolginov, 1975; Drobyshevski, 1975). Our general approach to the question of the impact hypothesis differs from that of the Soviet workers, for a variety of reasons, the most important of which has been our preoccupation with the observational results that we have assembled on CP stars over the past few years (cf. Cowley, 1975, 1976c). We shall therefore attempt an ab initio discussion of this question, setting out the strengths and weaknesses of the hypothesis as we see them, and outlining the general principles upon which the idea might be further developed or tested. It is worth pointing out that if the hypothesis is found to be of merit - and this is by no means implied - some light may be shed on the chemistry of the solar nebula and the planets.

There are, as we shall see, numerous objections which can immediately be raised to the hypothesis. However, none of the theories of $\mathrm{CP}$ stars is free from this kind of criticism, and the question of whether or not certain objections to one of the current theories are 'fundamental' or merely represent 'details to be worked out', is subject to tendentious reasoning.

It is now known that the early history of the solar system is replete with examples of collisions of solid bodies ranging up to planetary dimensions. Yet even in the case of the Moon, where the evidence for meteoritic impact is as strong as it could be, general recognition of this phenomena was a surprisingly long time coming.

A record of ubiquitous bombardment throughout the inner solar system is preserved on the surfaces of Mercury, Venus, Earth, Moon, and Mars (cf. Hartmann, 1977). It is not at all extraordinary, therefore, to suppose that the central object of a stellar

TABLE I

Masses relevant for the impact hypothesis

$\begin{array}{lr}\text { Mass of the photosphere of an A star } & \sim 3 \times 10^{22} \mathrm{gm} \\ \text { Mass of Fe, Mg, and Si in normal A star photosphere } & 7 \times 10^{19} \\ \text { Mass of rare-earth elements in normal A star photosphere } & 4 \times 10^{14} \\ \text { Mass of rare-earth elements in Ap star photosphere } & <4 \times 10^{19} \\ \text { Mass of Earth } & 6 \times 10^{27} \\ \text { Mass of Moon } & 7 \times 10^{25} \\ \text { Mass of the asteroid responsible for Imbrium Basin } & 10^{22} \\ \text { Mass of comet } & 10^{15}-10^{19}\end{array}$


system may have received its share of impacts. Indeed, if the evidence for such bombardment is entirely missing, it is a problem of no small interest to account for this fact.

In Table I we have collected some relevant masses for the impact hypothesis. If we are to take the hypothesis seriously at all, there must be sufficient mass in the impacting bodies to replace, as a minimum, the visible photosphere of an A star. The table shows that this consideration offers no immediate difficulty for the hypothesis.

In lower Main-Sequence stars, the roiling convection would soon distribute any added material throughout the stellar envelope, resulting in a small modification of the abundance. Joss (1974) has studied the accretion of cometary material and estimated that some $10^{26} \mathrm{~g}$ have been added to the Sun during its lifetime. With the stable envelope of an upper Main-Sequence star, one could reduce this mass estimate by many orders of magnitude and be left with a significant amount of material relative to the photospheric mass.

A marked change in the surface chemical composition due to impacts is not at all unreasonable.

A second problem of fundamental importance to the impact hypothesis concerns the time-scale for the formation of terrestrial planets and meteoroids. The MainSequence lifetime of an A0 star is $\sim 3 \times 10^{8} \mathrm{yr}$; for a B0 star it is $\sim 10^{7} \mathrm{yr}$. Is there enough time for the differentiated bodies to accumulate, before these upper MainSequence stars have exhausted their core hydrogen?

All aspects of the earliest phases of the history of the solar system are highly uncertain. We can only summarize here the ideas that have been expressed by workers in the field. Different hypotheses concerning the mode of planet formation lead, of course, to a variety of time-scales, but the visible record of impact on the surfaces of objects in the solar system implies that the bombardment was intense - perhaps most intense - near the time of solidification of the planetary surfaces. Thus, most theories of formation of the planets suggest formation over an interval of time that was significantly less than the age of the solar system; authorities quote time-scales of the order of $10^{8} \mathrm{yr}$ or less. Ringwood $(1975, \mathrm{p} .569)$ gives numerous references to estimates that range from $10^{3}$ to $10^{8} \mathrm{yr}$.

It therefore appears that the time-scale for the formation of bodies which are chemically highly differentiated need not pose a fundamental problem for the impact hypothesis. In our subsequent discussion, we shall assume that it does not.

\section{Physical Principals and Basic Data}

In order to confront the impact hypothesis with observations of stellar abundance patterns, we must have some means of deciding upon plausible compositions for the impacting bodies. Let us start by considering the Earth-Moon system.

We are immediately at a disadvantage. The chemical compositions of the terrestrial and lunar crusts are known fairly well, but the detailed compositions of the whole Earth and Moon are far more speculative. Some knowledge concerning the bulk compositions of the terrestrial planets is available from their densities. It is thus 
reasonable to infer that the Earth, Mercury, and Venus have a higher iron content than the Moon and Mars. Fascinating results on the composition of the Earth's core are available from seismic studies, and laboratory experiments on the propagation of sound waves in solid materials which have been subjected to transient pressures, comparable to those in the Earth's core, by means of shock waves. This work, however, is still subject to interpretation, and is unlikely to provide the detailed relative abundances of contiguous elements which are needed for a comparison with astronomical observations.

It has long been the practice to infer the bulk composition of the Earth from meteorites, and to apply such considerations as bulk density and seismic velocity as boundary conditions. Thus Mason (1966) assumed that the composition of the Earth's core was the same as that of the nickel-iron phases found in chrondrites. The mantle and crust were assumed to have a composition equivalent to the oxidized material in an 'average' chrondrite.

Certainly the meteorites are of tremendous value when we attempt to specify the composition of planetoids which might impact on stellar surfaces. Quite independent of the question of how well the composition of the Earth may be specified by some meteoric mixture, these objects obviously represent samples of solid cosmic material which have been extensively analyzed in the laboratory. It is, therefore, possible - with the help of meteoritic data - to subject the impact hypothesis to an immediate zero-order test: can we find a mixture of meteoritic material which, if added to the photosphere of an A star, would result in a composition similar to that which is observed in the CP stars? The answer to this question (see Section 4) appears to be 'No'.

Theoretical calculations have been made which predict condensation sequences in the solar nebula. With the help of such calculations, it is possible to understand, in a general way, the bulk composition of the planets and their satellites in terms of the physical conditions, primarily the temperature, in the nebula at the point of formation of planets (Barshay and Lewis, 1976). Calculations of this type might be used as a guide to the possible compositions of planetary bodies surrounding upper MainSequence stars for which the proto-planetary cloud might reasonably be expected to be hotter than the solar nebula. As we shall see, the general concept of temperaturedependent condensation sequences, combined with the impact hypothesis, leads us to expect that the abundance anomalies in the CP stars will be strongly temperature dependent.

The final principle that we shall mention as a means of evaluating the confrontation of the impact hypothesis with observation is the geochemical classification of the elements. The basic idea is due to Goldschmidt (cf. Goldschmidt, 1954), who divided the chemical elements into the categories listed in Table II. The classification is not precise, and some elements are listed in more than one category. Goldschmidt used both theoretical and empirical considerations in the classification. For example, the lithophiles vs. siderophiles are usually those elements for which the standard free 
TABLE II

Geochemical classification of the elements

\begin{tabular}{llll}
\hline Lithophile & Siderophile & Chalcophile & Atmophile \\
\hline $\mathrm{Li}, \mathrm{Be}, \mathrm{B}, \mathrm{O}, \mathrm{F}$, & $\mathrm{C}, \mathrm{P}, \mathrm{Fe}, \mathrm{Co}, \mathrm{Ni}$, & $\mathrm{S}, \mathrm{Cu}, \mathrm{Zn}, \mathrm{Ga}$, & $\mathrm{H}, \mathrm{N}, \mathrm{Noble}$ \\
$\mathrm{Na}, \mathrm{Mg}, \mathrm{Al}, \mathrm{Si}$, & $\mathrm{Ge}, \mathrm{Mo}, \mathrm{Ru}, \mathrm{Rh}$, & $\mathrm{As}, \mathrm{Se}, \mathrm{Ag}, \mathrm{Cd}$, & gases $(\mathrm{C}, \mathrm{O}, \mathrm{F}$, \\
$\mathrm{Cl}, \mathrm{K}, \mathrm{Ca}, \mathrm{Sc}, \mathrm{V}$, & $\mathrm{Pd}, \mathrm{Sn}, \mathrm{Re}, \mathrm{Os}$, & $\mathrm{Sb}, \mathrm{Te}, \mathrm{Hg}, \mathrm{Pb}$, & $\mathrm{Cl}, \mathrm{Br}, \mathrm{I})^{a}$. \\
$\mathrm{Cr}, \mathrm{Mn}, \mathrm{Br}, \mathrm{Rb}$, & $\mathrm{Ir}, \mathrm{Pt}, \mathrm{Au},(\mathrm{Pb}$, & $(\mathrm{Fe}, \mathrm{Ge}, \mathrm{Mo}, \mathrm{Re}$, & \\
$\mathrm{Sr}, \mathrm{Y}, \mathrm{Zr}, \mathrm{Nb}, \mathrm{I}$, & $\mathrm{As}, \mathrm{W})^{a}$. & $\mathrm{Au}, \mathrm{Sn})^{a}$. & \\
$\mathrm{Ca}, \mathrm{Ba}, \mathrm{Rare}$ & & & \\
earths, Hf, W & & & \\
$(\mathrm{H}, \mathrm{C}, \mathrm{N}, \mathrm{P}, \mathrm{Fe}$, & & \\
$\mathrm{Ga}, \mathrm{Ge}, \mathrm{Tl})^{a}$. & & & \\
\hline
\end{tabular}

${ }^{a}$ Elements listed in parentheses belong, at least partially, in other classes.

energies of formation of oxides $\left(\Delta F^{\circ}\right)$ are more negative than those of FeO. In a melt, or magma where both oxidized and reduced metallic iron are present, the lithophiles will form oxides, and, in the Earth, eventually silicates, and will be found primarily in the mantle and crust. The siderophiles prefer the reduced state. They have followed metallic iron and nickel into the Earth's core, so that they are now underabundant in the crust. The classification of elements into the chalcophile or sulfide-loving phase cannot be so readily explained in terms of the free energies of sulfide formation (cf. Rankama and Sahama, 1950). Here the classification rests almost entirely on observed associations. In meteorites, the chalcophiles are found as impurities in the mineral troilite (FeS).

Other classification schemes are now used, such as the one discussed by Ringwood (1975, p. 547). Ringwood drops the classifications lithophile and chalcophile and uses siderophile, refractory and volatile.

It is possible for the geochemical associations to mimic the predictions of nuclear processes, for example in the correspondence of the osmium-platinum-iridium $r$ process peak with the siderophile properties of these same elements. Caution is always advisable in extending a principle beyond the domain in which it has been established, but the general notion of geochemical associations is a potentially powerful tool with which to examine the impact hypothesis, and we shall use it below.

\section{Characteristic Properties of CP Stars and Impact Hypothesis}

In this section we shall point out how various properties of the CP stars may be explained in terms of the impact hypothesis.

1. It is well-known that the CP stars are slow rotators, and this observation has been difficult to connect with any of the nuclear theories of the abundance anomalies. In the diffusion hypothesis of $\mathrm{CP}$ anomalies, which requires a very stable atmosphere, it is supposed that rapid rotation causes mixing currents or turbulence which prevent chemical separation, and a correlation of abundance peculiarities with slow rotation is 
thus established. This has been considered one of the strong points of the diffusion hypothesis.

The slow rotation of the CP stars may also be naturally explained on the impact hypothesis. It is only necessary to suppose that a low value of the angular momentum per unit mass was also resident in the stellar nebula and the planets and planetesimals which formed from it. The low angular momentum will imply, cet. par., large eccentricities which will increase the probability of collision of the planetesimals with the central star.

2. There is a general relation between abundance anomalies in different families of $C P$ stars and stellar effective temperature. This relationship, which is quite well established observationally, has no simple explanation in terms of the nuclear theories of the origin of $\mathrm{CP}$ anomalies. The diffusion and magnetic accretion hypotheses appear to have much more potential for accounting for this observation. A straightforward interpretation is possible in terms of the impact hypothesis, since the bulk compositions of the planetoids which surround a star would be determined by the temperature in the stellar nebula (cf. Barshay and Lewis, 1976). We expect that the region of formation of terrestrial-type planets would be larger about hotter stars, and this would lead us to expect the abundance anomalies to increase with stellar effective temperature. There is some evidence that this is indeed the case (cf. Aller, 1970).

3. There are two sequences of CP stars, magnetic and non-magnetic and the abundance anomalies in the two sequences are more or less distinct. It has been repeatedly emphasized by Alfvén and his co-workers that the presence of a magnetic field will exert a strong influence on the conditions in a stellar nebula. Alfvén and Arrhenius (1975) discuss a most interesting mechanism for chemical differentiation in the solar nebula, based on the interaction of ions with a magnetic plasma that is co-rotating with the Sun. The idea is proposed as an alternate to the more conventional temperaturedependent condensation sequences. It is not necessary to accept the Alfvén-Arrhenius hypothesis in toto to imagine that the chemistry of a hydromagnetic stellar nebula could be quite different from that of a non-magnetic gas. Further discussion of chemical separation involving plasma-process is given by Arrhenius and Alfvén (1971), Lehnert (1976), and Bonnevier (1976).

4. The chemical peculiarities are correlated with the binary nature of the CP stars: The cooler metallic-line A stars are probably all binaries (Abt, 1961, 1967; see also Conti and Barker, 1973). The magnetic Ap stars show a lower than normal frequency of binaries, while the manganese stars appear to be normal in binary frequency (Abt and Snowden, 1973; Aikman, 1976). In a double-star system, it is easy to imagine that perturbations of planetoids leading to impact on stars would be more frequent than on single stars. In the case of the predominantly single magnetic CP stars, it could well be that the low angular momentum necessary for planetesimal impact can only be achieved if magnetic braking is present. It is necessary to suppose that the angular momentum of both the central star, and the stellar nebula are low because of magnetic interactions; this is not difficult. The chemical distinctions between the 
manganese stars and the magnetic Ap stars could be due to a combination of their temperatures, lack of magnetic fields, and normal binary frequency.

5. Chemical peculiarities occur in patches in magnetic stars and are uniformly distributed on the surfaces of non-magnetic CP stars. The impact hypothesis accounts in a natural way for surficial abundance inhomogeneities. In order to explain the observations it is necessary to assume that the magnetic field preserves the patches while in the absence of a field the dissolution of a spot can take place on a time-scale short with respect to the lifetime of the star. This problem has been recently studied by Havnes (1975b), who concludes that horizontal diffusion in a magnetic field will damp composition differences in older Ap stars but will not produce completely homogeneous surfaces. In non-magnetic regions of Ap stars, or on non-magnetic stars, more efficient mixing mechanisms might produce uniform surfaces. We find this quite plausible since horizontal mixing would probably involve velocities of the order of the observed microturbulent motions, i.e., velocities of the order of a kilometer per second, while the diffusion velocities calculated by Havnes are $<2 \times 10^{-4} \mathrm{~cm} \mathrm{~s}^{-1}$. There are thus some nine orders of magnitude between the estimates of horizontal mixing in magnetic and non-magnetic stars, which appears to be quite sufficient to account for the lack of spectral variations in the latter.

One rather puzzling observational result is the fact that abundance peculiarities in the patches show no definite relationship. In $\alpha^{2} \mathrm{CVn}$, for example, Fe II and Eu II are in antiphase; they are in phase in HR 5355 (Deutsch, 1956). The association of particular abundance anomalies with magnetic geometry is also unclear. The europium lines usually reach a maximum at a magnetic pole but there is no preference for positive or negative polarity, or for the strong rather than the weak pole. The impact hypothesis provides a natural basis for such vicissitude.

6. A great variety is observed among the abundance patterns in the CP stars. This writer feels it is possible that when a sufficiently wide sample of CP stars has been studied, it will be found that the different classes will merge smoothly into one another, denuding the classifications of meaning in terms of distinct physical mechanisms. This extreme position is not universally held, but all spectroscopists with detailed experience at high dispersion agree that important chemical distinctions occur within each of the broad divisions of the CP stars.

This variety in the chemical composition confounds all theories which have been proposed to date. For virtually every theoretical prediction of an abundance anomaly, the observer can point to an exception. Such a situation does not necessarily mean that the proposed physical mechanisms are invalid. But it does imply that the current theories are not sufficiently complex. The observations demand that contingency be an integral part of any successful theory of the CP stars.

The impact hypothesis provides amply for varied abundance patterns. Contingency is present at all phases of the mechanism from the chemical differentiation of terrestrial-type planets to the perturbations which ultimately make them collide with the central star. It may well be that current versions of the diffusion theory can be 
developed to give the observed complexity of $\mathrm{CP}$ abundances, but at the present time the ability to produce a variety of abundance patterns is a strong point in favor of the impact hypothesis.

7. Many of the arguments that support other differentiation hypotheses also apply to the impact hypothesis. For example:

(a) The hypotheses provide only surficial anomalies. It is a popular notion that the $\mathrm{CP}$ anomalies cannot apply throughout the bulk of the star. The basis of this idea perhaps requires re-examination in the light of recent work, which shows that $\mathrm{CP}$ stars do not necessarily fall on unevolved Main Sequences in clusters (Hartoog, 1976; Pendl and Seggeweiss, 1976).

(b) A stable photosphere is necessary to all mechanisms.

(c) Diffusion would support abundance anomalies caused by impacts.

(d) The peculiarities can arise in unevolved stars.

(e) The general results of all hypotheses are an enhancement of heavy elements, relative to certain of the lighter ones: $\mathrm{He}, \mathrm{C}, \mathrm{N}$ and $\mathrm{O}$.

\section{Weaknesses of the Impact Hypothesis}

A principal weakness of the impact hypothesis is its difficulty in producing a deficiency in the ratio of the abundance of any element relative to hydrogen. This statement presupposes that the stellar material contains normal abundances of all elements relative to hydrogen before the impact events, and that no significant sources of continuous opacity are added. If material were added to the photosphere of a star, the lines due to the added elements would strengthen, but in the first approximation there would be no change in the strengths of lines due to elements which were not added by the impact.

This conflicts with the well-established weakness of certain of the lighter elements in the CP stars. Underabundances of helium and oxygen occur in the magnetic Ap stars, while in the metallic-line A stars, calcium and scandium are typically underabundant.

The only means of surmounting this difficulty is to suppose the impacting planets carry with them a significant source of opacity. At the temperature of an A star, it is unlikely that there is any unknown source of opacity that could compete with hydrogen, and we are left with the problem of adding large quantities of hydrogen without adding either helium or oxygen.

Helium is a chemically inert gas, and it is not beyond a possibility that planetesimals could form in a region of a stellar nebula that was sufficiently cool for hydrogen to condense (perhaps in various ices) but not cool enough for helium. If these planetesimals found their way to the surface of a star, they could reduce the helium-tohydrogen ratio. Our picture, then, would involve the impact of a massive helium-poor planet rather than a terrestrial one, and this would almost certainly mean that the lighter elements other than helium would be normal or overabundant with respect to 
hydrogen. Thus, in the extreme case that $\mathrm{H}_{2}$ gas condensed but not $\mathrm{He}$, we would at best have an object with a normal oxygen-to-hydrogen ratio. Oxygen is very active chemically, and it is inconceivable that it could be lost from a region of a nebula in which hydrogen was condensing.

The mechanism of chemical differentiation proposed by Alfvén and Arrhenius (1975) appears to be capable of providing a hydrogen rich, outermost layer of a star. Indeed, one of the principal objections to their theory as a mechanism of chemical differentiation in the solar nebula is a strong point in favor of these ideas in the present context (Alfvén and Arrhenius, 1975, p. 229, 'objection (c)'), since it would produce a hydrogen-rich region close to the star. Briefly, their mechanism works as follows: a magnetic star is surrounded by a thin, co-rotating plasma. A neutral, low density gas will move freely through this plasma until its atoms reach a critical velocity

$$
v_{\text {crit }}=\sqrt{\frac{2 \chi_{1}}{m}},
$$

where $\chi_{\mathrm{i}}$ is the ionization energy and $m$ is the mass of the atom. The theoretical basis for this critical velocity is complex (Sherman, 1973), but its existence is well rooted in experimental work (Danielsson, 1973).

If the particles fall freely toward a proto-star of mass $M$, the critical velocity will be reached at a distance $r$ such that

$$
\frac{1}{2} m v_{\text {crit }}^{2}=\frac{G M m}{r}=\chi_{1}
$$

Hydrogen, with its low mass and high ionization potential would approach the star more closely than any of the other elements.

The relevance of these ideas is moot. For a $2 M_{\odot}$ star, we find from Equation (2), $r=2.0 \times 10^{13} \mathrm{~cm}$ for hydrogen and $4.5 \times 10^{13}$ for helium, so that the separation would be taking place at rather large distances from the surface of a Main-Sequence A star. There is, further, the problem of removal of the residual gas which has been depleted in hydrogen. If this were to be accomplished by a wind from the surface of the star, the hydrogen-enriched surficial gas could be lost again.

We conclude that observed underabundances in CP stars are still a formidable difficulty for the impact hypothesis.

Recent analyses of CP atmospheres accentuate this difficulty for the impact hypothesis. Abundances determined prior to the revision of the $g f$-value scale for iron (and other iron peak elements) found typical enhancements of the iron peak by factors of two to ten. Indeed, at one time (cf. the review by Conti, 1970) it was considered to be useful to define the Am stars in terms of an overabundance of iron-peak elements, as well as an underabundance of $\mathrm{Ca}$ (and $\mathrm{Sc}$ ) which characterize the spectroscopic appearance of these stars.

Allen (1976) has studied 18 normal and CP stars, and finds that iron sometimes departs significantly from the canonical value of $7.4(\log H=12.0)$. However, some 
highly peculiar stars, specifically $\gamma$ Equ and 53 Tau, show iron deficiencies. It has been unclear for some time - and perhaps it still remains unclear - whether one can take stellar abundance differences seriously, unless they are larger than an order of magnitude, and this is not yet the case with iron in $\gamma$ Equ and 53 Tau, where the deficiencies were found to be 0.2 and 0.6 dex. However, if one looks at the basic data for these stars - the line strengths, and compares them with line strengths in 'normal' MainSequence stars having similar effective temperatures, it is clear that the iron lines in $\gamma$ Equ and 53 Tau are weaker. No conceivable non-LTE ionization phenomena could be responsible for this result which is so well documented by lines in two stages of ionization.

We conclude that a simultaneous overabundance of manganese (and other elements) along with a deficiency in iron is a genuine difficulty for the impact hypothesis.

According to the analyses reported by Goles (1971), manganese is generally very low in the metal phases of iron meteorites. The $\mathrm{Fe} / \mathrm{Mn}$ ratio must be at least $10^{4}-10^{5}$. Under extreme reducing conditions (i.e., we postulate an oxygen deficiency), one might imagine that virtually all of the iron, in some hypothetical differentiated planet went into the core, leaving a mantle highly enriched in manganese, and other elements whose affinity for oxygen was greater than that of iron. Such elements would be the traditional lithophiles.

It would then be necessary to imagine that the core and mantle of this planet were separated, by a catastrophe of the kind that has given rise to separate stony and iron meteorites, and the material from the mantle made its way to the stellar photosphere.

Geochemical difficulties with this line of reasoning are immediate. The manganese stars are frequently enriched in platinum which is siderophile, and should have followed the iron into the core. Likewise, the elements chromium and titanum should be enhanced by factors similar to that of manganese since their lithophile character is even more marked. Now it is true that enhanced chromium is a common occurrence in Ap stars, but not necessarily in those Ap stars that are enriched in manganese. Allen and Cowley (1976) find that $\mathrm{Cr}$ is normal in $\gamma \mathrm{Equ}$, which shows Mn enhanced. $\mathrm{Cr}$ is also normal or weak in HR 7664, for which Mn is enhanced by $1.3 \mathrm{dex}$.

Dworetsky (1971) has reviewed abundance work on the mercury-manganese stars, and has made the very important adjustment of the results for the revision of the $g f$ value scale for iron and selected iron group elements. This compilation is based primarily on the extensive abundance studies made by Lawrence Aller and his students at UCLA in the 1960's. The results, after the correction for the $g f$-scales, are in remarkably good agreement with those of Allen (1976). In particular, the enormous enhancements of the siderophile platinum in stars with normal or marginally enhanced iron is confirmed.

Following Bidelman's (1962) identification of $\mathrm{Hg} \lambda 3984$ in the manganese stars, the study of this element has been pursued with keen interest. Recent discussions have been made by Cowley and Aikman (1975) and White et al. (1976). The original identification was made on the basis of one line, a rather courageous step, but when 
the investigation of several stars showed the rest wavelength to be variable, Bidelman had the audacity to suggest that the wavelength shifts were due to changing isotopic abundances of mercury.

We must refer to the papers which we have cited for an account of how well Bidelman's suggestions have stood the test of time - and withstood the scrutiny of numerous spectroscopists, this writer among them. Today, the 'mercury question' is one of the most perplexing of the CP star abundance anomalies.

Mercury is traditionally classed, geochemically, as a chalcophile, but in the more recent classifications such as that of Ringwood (1975) it is described as a 'volatile', along with a number of other chalcophile elements such as $\mathrm{Zn}$ and $\mathrm{Cd}$. These chalcophile-volatile elements are depleted in the chrondrites relative to the carbonaceous chondrites. In the Earth's and Moon's crust both chalcophile and siderophile elements are depleted. We do not really know whether the chalcophiles are underabundant in the terrestrial and lunar crusts because they followed the siderophiles into the core, or whether they were lost prior to accretion. If volatility is the dominant property, we would not expect mercury to be overabundant at all, while if association with an $\mathrm{Fe}-\mathrm{FeS}$ core dominates, we would expect to find iron and mercury to be enhanced together.

Reed (1971) tabulates determinations in the ordinarily uniform Type I carbonaceous chondrites which vary by a factor of 100 .

It is very difficult to associate any of these ideas with the observed stellar abundances except to note that all are subject to vicissitude.

Dworetsky et al. (1970) showed that the mercury abundance in the star $\chi$ Lup was dominated by the heaviest isotope ${ }^{204} \mathrm{Hg}$. They estimated that $97 \%$ of the $\mathrm{Hg}$ was ${ }^{204} \mathrm{Hg}, 3 \%{ }^{202} \mathrm{Hg}$, and did not give values for the lighter isotopes. Such severe isotopic fractionation is unknown in geochemistry, which measures the deviation $\delta$ in isotopes from standard abundances in parts per thousand. This writer is unable to think, even, of an implausible scheme by which the impact hypothesis could account for this observational result.

Michaud et al. (1974) have proposed a scheme in which the lighter mercury isotopes are pushed out of the photospheres of the manganese stars. Kuchowicz (1975) notes that an $r$-process would lead naturally to an enrichment of the heavier mercury isotopes. Both of these ideas need to be explored in terms of predictions which the mechanisms make for abundances of other elements. Neither has been widely accepted, and the mercury isotope question remains as puzzling as ever.

In the hottest chemically peculiar stars, the dominant helium isotope is sometimes observed to be ${ }^{3} \mathrm{He}$ (see, for example, the discussion by Bashek, 1975). It has been very difficult to account for this observation in terms of standard nuclear processes and stellar evolution, although some schemes have been suggested. Michaud and Vauclair (1972), and Vauclair et al. (1974) have proposed a diffusion mechanism to explain the enhancement of ${ }^{3} \mathrm{He} /{ }^{4} \mathrm{He}$. Here, we note that the Alfvén-Arrhenius mechanism is also capable of producing a separation of ${ }^{3} \mathrm{He}$ and ${ }^{4} \mathrm{He}$. Equation (2) shows that the 
distance at which ${ }^{3} \mathrm{He}$ would reach $v_{\text {crit }}$ is less by $25 \%$ than that for ${ }^{4} \mathrm{He}$. This mechanism has not yet been explored quantitatively, and at this point the variable helium isotopes pose as grave difficulties for the impact hypothesis as the 'mercury problem'.

\section{Conclusions}

In this paper we have seen that the impact of planetesimals on the surface of an upper Main-Sequence star is capable of a substantial alteration of the surficial composition. Many of the properties of the CP stars are in good accord with the premises upon which the impact hypothesis is based.

The difficulties that we have mentioned in the preceding section preclude the impact hypothesis as the sole mechanism for all CP star abundance peculiarities. However, this does not mean that the entire set of ideas that we have reviewed can be dismissed out of hand.

The prospects for the impact hypothesis are brightest if it is allowed to work in synergism with one of the other CP star mechanisms, such as diffusion. This could readily supply the extra degrees of freedom that the observations require.

The question of whether these mechanisms of chemical differentiation can remove the entire burden of the CP phenomena is still moot. The mercury isotopic abundances and the uranium-to-thorium ratio (Cowley et al., 1976) still hint strongly, in our opinion, of nuclear processes. A much more detailed concordance of observational results with theoretical calculations is necessary before any of the hypotheses concerning CP stars can be definitely rejected.

\section{Acknowledgements}

It is a pleasure to thank Acting Director E. H. Richardson for the use of the facilities of the Dominion Astrophysical Observatory. G. C. L. Aikman and A. H. Batten have been kind enough to read an early version of the manuscript and offer a number of useful comments. I also wish to thank the Department of Physics of the University of Victoria for a visiting professorship, and for assistance in the preparation of this manuscript for publication. This research was supported by the National Science Foundation.

\section{References}

Abt, H.: 1961, Astrophys. J. Suppl. 51, 37.

Abt, H.: 1967, in R. C. Cameron (ed.), The Magnetic and Related Stars, Mono Book Corp., Baltimore.

Abt, H. and Snowden, M.: 1973, Astrophys. J. Suppl. 25, 137.

Aikman, G. C. L.: 1976, Publ. Dom. Astrophys. Obs. 14, 379.

Alfvén, H. and Arrhenius, G.: 1975, Structure and Evolutionary History of the Solar System, D. Reidel Publ. Co., Dordrecht. 
Allen, M. S.: 1976, Ph.D. thesis, University of Michigan.

Allen, M. S. and Cowley, C. R.: 1976, Bull. Am. Astron. Soc. 8, 522.

Aller, M. F.: 1970, Astron. Astrophys. 6, 67.

Arrhenius, G. and Alfvén, H.: 1971, Earth Planetary Sci. Lett. 10, 253,

Barshay, S. S. and Lewis, J. S.: 1976, Ann. Rev. Astron. Astrophys. 14, 81.

Bashek, B.: 1975, in B. Bashek, W. H. Kegel, G. Traving (eds.), Problems in Stellar Atmospheres and Envelopes, Springer-Verlag, Berlin, p. 101.

Bidelman, W. P.: 1962, Astron. J. 67, 645.

Bonnevier, B.: 1976, Astrophys. Space Sci. 40, 231.

Conti, P. S.: 1970, Publ. Astron. Soc. Pacific 82, 781.

Conti, P. S. and Barker, P. K.: 1973, Astrophys. J. 186, 185.

Cowley, C. R.: 1975, in W. W. Weiss, H. Jenkner, and H. J. Wood (eds.), Physics of Ap Stars, IAU Colloq. No. 32, Finsterle and Co., Vienna, p. 275.

Cowley, C. R.: 1976a, Sky Telesc. 52, 236.

Cowley, C. R.: 1976b, Sky Telesc. 52, 341.

Cowley, C. R.: 1976c, Astrophys. J. Suppl. 32, 631.

Cowley, C. R. and Aikman, G. C. L.: 1975, Publ. Astron. Soc. Pacific 87, 513.

Cowley, C. R., Allen, M. S., and Aikman, G. C. L.: 1976, Astrophys. Lett. 18, 83.

Danielsson, L.: 1973, Astrophys. Space Sci. 24, 459.

Deutsch, A. J.: 1956, Publ. Astron. Soc. Pacific 68, 92.

Dolginov, A. Z.: 1975a, in W. W. Weiss, H. Jenkner, and H. J. Wood (eds.), Physics of Ap Stars, IAU Colloq. No. 32, Finsterle and Co., Vienna, p. 752.

Drobyshevsky, E. M.: 1975, Astrophys. Space Sci. 35, 403.

Dworetsky, M. M.: 1971, Ph.D. Thesis, University of California at Los Angeles.

Dworetsky, M. M., Ross, J. E., and Aller, L. H.: 1970, Bull. Am. Astron. Soc. 2, 311.

Goldschmidt, V. M.: 1954, Geochemistry, University Press, Oxford.

Goles, G. G.: 1971, in B. Mason (ed.), Handbook of Elemental Abundances in Meteorites, Gordon and Breach, New York, p. 201.

Guthrie, B. N. G.: 1971, Astrophys. Space Sci. 13, 168.

Hartmann, W. K.: 1977, Sci. Amer. 236, 84.

Hartoog, M. R.: 1976, Astrophys. J. 205, 807.

Havnes, O.: 1975a, in W. W. Weiss, H. Jenkner, and J. G. Wood (eds.), Physics of Ap Stars, IAU Colloq. No. 32, Finsterle and Co., Vienna, p. 135.

Havnes, O.: 1975b, Astron. Astrophys. 38, 105.

Havnes, O. and Conti, P. S.: 1971, Astron. Astrophys. 14, 1.

Jaschek, M. and Jaschek, C.: 1974, Vistas Astron. 16, 131.

Joss, P. C.: 1974, Astrophys. J. 191, 771.

Kuchowicz, B.: 1975, in W. W. Weiss, H. Jenkner, and H. J. Wood (eds.), Physics of Ap stars, IAU Colloq. No. 32, Finsterle and Co., Vienna, p. 169.

Lehnert, B.: 1976, Astrophys. Space Sci. 40, 225.

Mason, B.: 1966, Principles of Geochemistry, 3rd ed., John Wiley and Co., New York.

Michaud, G.: 1970, Astrophys. J. 160, 641.

Michaud, G.: 1975, in W. W. Weiss, H. Jenkner, and H. J. Wood (eds.), Physics of Ap Stars, IAU Colloq. No. 32, Finsterle and Co., Vienna, p. 81.

Michaud, G. and Vauclair, S.: 1972, Astrophys. Lett. 11, 117.

Michaud, G., Reeves, H., and Charland, Y.: 1974, Astron. Astrophys. 37, 313.

PendI, E. S. and Seggeweiss, W: 1975, in W. W. Weiss, H. Jenkner, and H. J. Wood (eds.), Physics of Ap Stars, IAU Colloq. No. 32, Finsterle and Co., Vienna, p. 357.

Preston, G. W.: 1974, Ann. Rev. Astron. Astrophys. 12, 257.

Rankama, K. and Sahama, Th. G.: 1950, Geochemistry, The University of Chicago Press, Chicago, p. 91.

Reed, Ge W., Jr.: 1971, in B. Mason (ed.), Handbook of Elemental Abundances in Meteorites, Gordon and Breach, New York, p. 487.

Ringwood, A. E.: 1975, Composition and Petrology of the Earth's Mantle, McGraw Hill, New York. 
Sargent, W. L. W.: 1964, Ann. Rev. Astron. Astrophys. 2, 297.

Sherman, J. C.: 1973, Astrophys. Space Sci. 24, 487.

Vauclair, S., Michaud, G., and Charland, Y.: 1974, Astron. Astrophys. 31, 381.

White, R. E., Vaughan, A. H. Jr., Preston, G. W., and Swings, J. P.: 1976, Astrophys. J. 204, 131. 